\title{
VLT/X-shooter spectroscopy of the GRB 090926A afterglow ${ }^{\star}$
}

\author{
V. D’Elia ${ }^{1,2}$, J. P. U. Fynbo ${ }^{3}$, S. Covino ${ }^{4}$, P. Goldoni ${ }^{5,6}$, P. Jakobsson ${ }^{7}$, F. Matteucci ${ }^{8}$, S. Piranomonte ${ }^{1}$, J. Sollerman ${ }^{3,9}$, \\ C. C. Thöne ${ }^{4}$, S. D. Vergani ${ }^{10,11}$, P. M. Vreeswijk ${ }^{3}$, D. J. Watson ${ }^{3}$, K. Wiersema ${ }^{12}$, T. Zafar ${ }^{3}$, A. de Ugarte Postigo ${ }^{4}$, \\ H. Flores ${ }^{11}$, J. Hjorth ${ }^{3}$, L. Kaper ${ }^{13}$, A. J. Levan ${ }^{14}$, D. Malesani ${ }^{3}$, B. Milvang-Jensen ${ }^{3}$, E. Pian ${ }^{8,15}$, \\ G. Tagliaferri ${ }^{4}$, and N. R. Tanvir ${ }^{12}$
}

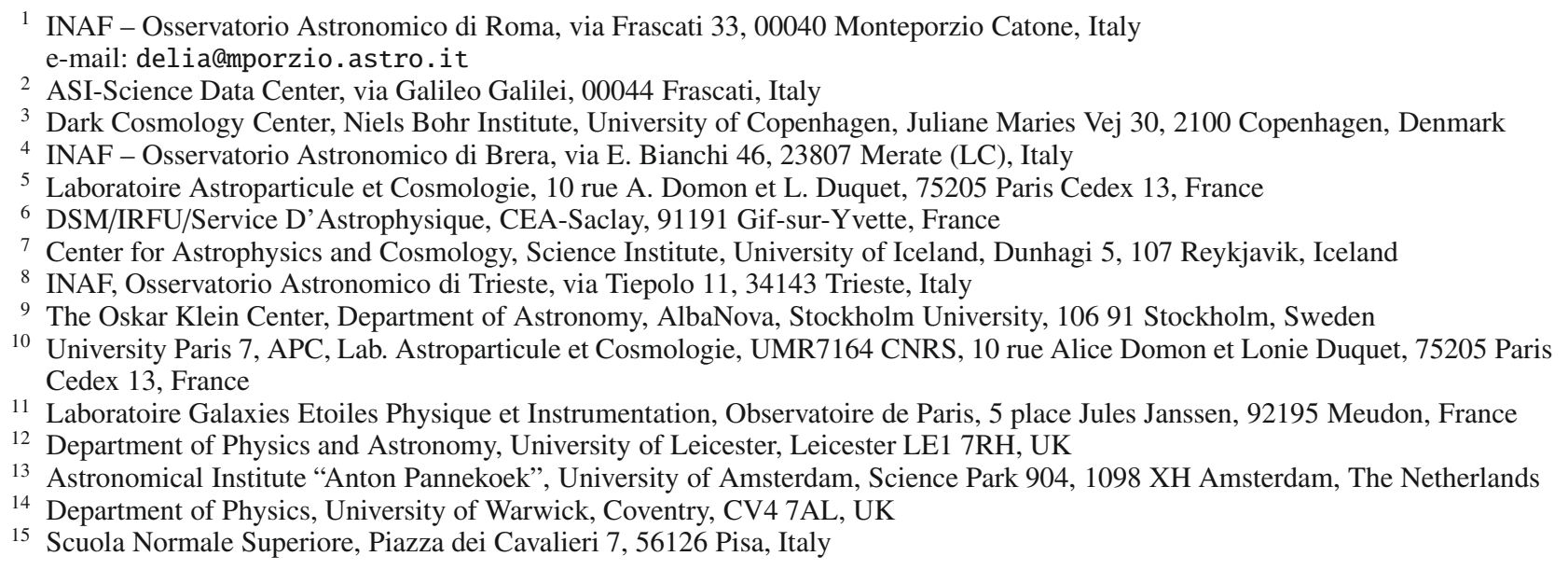

Received 15 June 2010 / Accepted 29 July 2010

\section{ABSTRACT}

\begin{abstract}
Aims. The aim of this paper is to study the environment and intervening absorbers of the gamma-ray burst GRB 090926A through analyzing optical spectra of its afterglow.

Methods. We analyzed medium-resolution spectroscopic observations $\left(R=10000\right.$, corresponding to $30 \mathrm{~km} \mathrm{~s}^{-1}, S / N=15-30$ and wavelength range 3000-25 000) of the optical afterglow of GRB 090926A, taken with X-shooter at the VLT $22 \mathrm{~h}$ after the GRB trigger.

Results. The spectrum shows that the ISM in the GRB host galaxy at $z=2.1071$ is rich in absorption features, with two components contributing to the line profiles. In addition to the ground state lines, we detect C II, O I, Si II, Fe II, and Ni II-excited absorption features, which we used to derive information on the distance between the host absorbing gas and the site of the GRB explosion. The distance of component I is found to be $2.40 \pm 0.15 \mathrm{kpc}$, while component II is located far away from the GRB, possibly at $\sim 5 \mathrm{kpc}$. These values are compatible with those found for other GRBs. The hydrogen column density associated to GRB 090926A is $\log N_{\mathrm{H}} / \mathrm{cm}^{-2}=21.60 \pm 0.07$, and the metallicity of the host galaxy is in the range $[\mathrm{X} / \mathrm{H}]=-2.5$ to -1.9 with respect to the solar values, i.e., among the lowest values ever observed for a GRB host galaxy. A comparison with galactic chemical evolution models has suggested that the host of GRB090926A is likely to be a dwarf-irregular galaxy. No emission lines were detected, but a H $\alpha$ flux in emission of $9 \times 10^{-18} \mathrm{erg} \mathrm{s}^{-1} \mathrm{~cm}^{-2}$ (i.e., a star-formation rate of $2 M_{\odot} \mathrm{yr}^{-1}$ ), which is typical of many GRB hosts, would have been detected in our spectra, and thus emission lines are well within the reach of $\mathrm{X}$-shooter. We put an upper limit to the $\mathrm{H}$ molecular fraction of the host galaxy ISM, which is $f<7 \times 10^{-7}$. The continuum has been fitted assuming a power-law spectrum, with a spectral index of $\beta=0.89_{-0.02}^{+0.02}$. The best fit does essentially not require any intrinsic extinction because $E_{B-V}<0.01$ mag adopting a SMC extinction curve. Finally, the line of sight of GRB 090926A presents four weak intervening absorption systems in the redshift range $1.24<z<1.95$.
\end{abstract}

Key words. ISM: abundances - ISM: atoms - galaxies: star formation - line: profiles - techniques: spectroscopic - atomic processes

\section{Introduction}

The study of the interstellar medium (ISM) of $z \gtrsim 1$ galaxies has traditionally relied upon Lyman-break galaxies (LBGs) at $z=3-4$ (see e.g. Steidel et al. 1999), $K$-band selected galaxies (Savaglio et al. 2004), and galaxies that happen to be along the

* Based on observations collected at the European Southern Observatory, ESO, the VLT/Kueyen telescope, Paranal, Chile, during the science verification phase, proposal code: 060-9427(A). lines of sight to bright background quasars (or QSOs). However, LBGs are characterized by pronounced starformation and their inferred chemical abundances may relate to these regions rather than representing typical high-redshift galaxies. Weak metal line systems along the line of sight to quasars probe mainly galaxy halos, rather than their bulges or discs (Fynbo et al. 2008). Taking advantage of ultra-deep Gemini multi-object spectrograph observations, Savaglio et al. $(2004,2005)$ studied the ISM of a sample of faint $K$-band selected galaxies at $1.4<z<2.0$, 
finding MgII and FeII abundances much higher than in QSO systems but similar to those in GRB host galaxies. Such studies can hardly be extended to higher redshift with the present generation of $8 \mathrm{~m}$ class telescopes, because of the faintness of high-redshift galaxies. Since the discovery that gamma-ray bursts (GRBs) are extragalactic, we now can avail ourselves of an independent tool to study the ISM of high-redshift galaxies.

Metallicities measured in GRB host galaxies vary from less than $10^{-2}$ to nearly solar values and are on average higher than those found along QSO sightlines (see e.g., Fynbo et al. 2006; Prochaska et al. 2007). This result supports the notion that GRBs originate in dwarf and/or low-mass galaxies. Since star formation occurs in molecular clouds, the latter are expected to be the GRB birthplaces. In this scenario, absorption from groundstate and vibrationally excited levels of the $\mathrm{H}_{2}$ molecules are expected, but these are typically not observed in GRB afterglow spectra (Vreeswijk et al. 2004; Tumlinson et al. 2007). The nondetection of $\mathrm{H}_{2}$ molecules (with the exception of GRB 080607 , see Prochaska et al. 2009; Sheffer et al. 2009) can possibly be explained by photo-dissociation by the intense UV flux from the GRB afterglow.

Indeed, the main difference between QSO and GRB absorption spectroscopy is that QSOs are nearly stationary in their emission, while GRBs are the most variable and violent phenomena in the Universe. Thus, while QSOs have the time to ionize the ISM along their lines of sight, the physical, dynamical and chemical status of the circumburst medium in the star-forming region hosting GRB progenitors can be modified by the explosive event, through shock waves and ionizing photons. The transient nature of GRBs is manifested through the detection of fine structure and other excited levels of the atom O I and the ions Fe II, Ni II, Si II and C II. These features are routinely identified in GRB spectra, and are most probably excited by the intense UV flux from the afterglow, since strong variation is observed when multi-epoch spectroscopy is available. This variation is not consistent with a pure infrared excitation or collisional processes (Prochaska et al. 2006; Vreeswijk et al. 2007; D'Elia et al. 2009a). Thus, assuming UV pumping is the responsible mechanism for the production of these lines, the distance of the gas to the GRB can be computed. This distance turns out to be a few hundred pc (see D'Elia et al. 2009b, for GRB 080330 and Ledoux et al. 2009, for GRB 050730) or even in the kpc scale (see Vreeswijk et al. 2007, for GRB 060418 and D'Elia et al. 2009a, for the naked-eye GRB 080319B).

GRB spectroscopy is also suitable for studying systems lying along the line of sight to GRBs. Surprisingly, the number density of strong Mg II intervening absorbers in GRB spectra is more than twice higher than along QSO sightlines (Prochter et al. 2006; Vergani et al. 2009), while CIV absorbers do not show any statistical difference (Sudilovsky et al. 2007; Tejos et al. 2007). The reason for the Mg II excess in GRB spectra is still unclear, and a larger sample is needed to properly address this issue (Porciani et al. 2007; Cucchiara et al. 2009).

All these issues can now be systematically addressed using the X-shooter spectrograph. This is the first second-generation instrument at the ESO's very large telescope (VLT) at Paranal Observatory (Chile). It is a single target-spectrograph capable of obtaining a medium-resolution spectrum $(R=\lambda / \Delta \lambda=$ 4000-14 000) covering the spectral range 3000-24800 $\AA$ in a single exposure thanks to splitting the light into three arms: ultraviolet/blue (UVB), visual (VIS), and near-infrared (NIR). The high spectrograph efficiency allowed us to obtain good quality observations of the GRB 090313 afterglow (de Ugarte Postigo et al. 2010), although it was observed two days after the burst and
Table 1. X-shooter observations.

\begin{tabular}{lccc}
\hline \hline Observation & Time since burst $(\mathrm{h})$ & Exposure $(\mathrm{s})$ & $S / N$ \\
\hline 1 & 22.13 & 600 & $5-15$ \\
2 & 22.29 & 600 & $5-15$ \\
3 & 22.51 & 600 & $5-15$ \\
4 & 22.68 & 600 & $5-15$ \\
$1+2+3+4$ & 22.37 & 2400 & $10-30$ \\
\hline
\end{tabular}

under unfavorable conditions. We refer the reader to D'Odorico et al. (2006) for a more complete description of the X-shooter specifications and capabilities.

We discuss here the case of GRB 090926A, observed by $\mathrm{X}$-shooter on 27 September 2009. We investigate both the local medium surrounding the GRB and the intervening systems. We derive the metallicity by comparing the column densities of Hydrogen and metals, we search for molecular absorption and galactic emission lines, and we constrain the distance between the GRB and the absorber by comparing the ratios between the ground- and excited-level column densities with photoexcitation codes, under the assumption of indirect UV pumping production of the excited levels. An analysis of the intervening absorbers lying along the line of sight to the GRB afterglow and a search for diffuse interstellar bands (DIBs) is also presented.

The paper is organized as follows. Section 2 makes a short summary of the GRB 090926A detection and observations from the literature; Sect. 3 presents the X-shooter observations and data reduction; Sects. 4 and 5 are devoted to the study of the features from the host galaxy; Sect. 6 derives the extinction curve shape for this GRB; Sect. 7 presents the analysis of the other absorbing systems identified in the GRB 090926A line of sight; finally in Sect. 8 the results are discussed and conclusions are drawn. We assume a cosmology with $H_{0}=70 \mathrm{~km} \mathrm{~s}^{-1} \mathrm{Mpc}^{-1}$, $\Omega_{\mathrm{m}}=0.3, \Omega_{\Lambda}=0.7$. Hereafter, the [] refers to element abundances relative to solar values and $\mathrm{X}$ refers to any chemical element.

\section{GRB 090926A}

GRB 090926A was discovered by Fermi on September 26, 2009, at 04:20:26 UT, and was detected by both the GBM (Bissaldi 2009) and the LAT (Uehara et al. 2009) instruments. Swift pointed at the target $13 \mathrm{~h}$ later and found the X-ray counterpart (Vetere et al. 2009). The afterglow was later reported to be detected at optical wavelengths with the Skynet/PROMPT telescopes (Haislip et al. 2009) and with UVOT (Gronwall \& Vetere 2009), the reported PROMPT magnitude was $R \sim 18$, although the observations took place almost $20 \mathrm{~h}$ after the burst. The redshift was secured by X-shooter, which observed the afterglow $2 \mathrm{~h}$ after these optical detections. The preliminary reported value was $z=2.1062$ (Malesani et al. 2009). FORS2 spectroscopic observations and GROND photometric follow-up of the GRB 090926A afterglow have been presented in Rau et al. (2010, hereafter R10), and we compare our results with theirs in the next sections.

\section{Observations and data reduction}

In the framework of the science verification phase program, we observed the afterglow of GRB 090926A with X-shooter (D'Odorico et al. 2006), mounted at the VLT-UT2 telescope. The observations consist of 4 different exposures of $600 \mathrm{~s}$ each (see Table 1). The exposures were taken using the technique of 

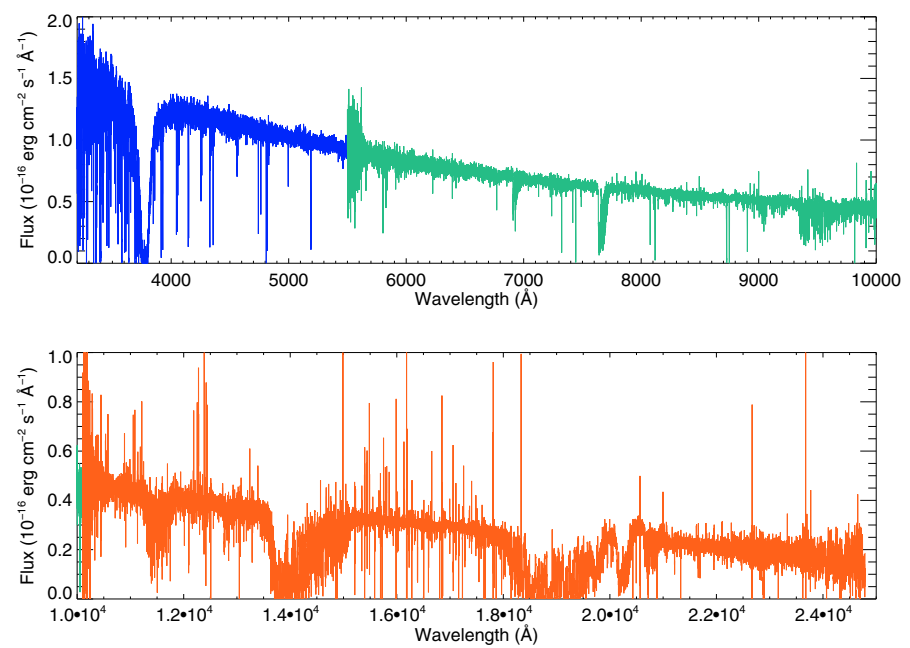

Fig. 1. The flux-calibrated X-shooter spectrum of GRB 090926A. Top: ultraviolet/blue and visual arms. Bottom: near-infrared arm. The absolute flux calibration may be not accurate due to slit losses.

nodding along the slit with an offset of 5 arcsec between exposures in a standard ABBA sequence. The sequence began on September 27, 2007 at 02:23:14 UT, $22 \mathrm{~h}$ after the GRB trigger. The magnitude of the afterglow at the time of the observation was reported by $\mathrm{R} 10$ to be $R=18.7$, which translates into a $6600 \AA$ flux of $0.8 \times 10^{-16} \mathrm{erg} \mathrm{cm}^{-2} \mathrm{~s}^{-1} \AA^{-1}$. The total net exposure time of our observations is $40 \mathrm{~min}$. The slit width was set to $0.9^{\prime \prime}$ in the VIS and NIR arms and $1.0^{\prime \prime}$ in the UVB arm. The UVB and VIS CCD detectors were rebinned to $1 \times 2$ pixels (binned in the spectral direction but not in the spatial one) to reduce the readout noise.

We processed the spectra using a preliminary version of the $\mathrm{X}$-shooter data reduction pipeline (Goldoni et al. 2006). The pipeline performed the following actions: The raw frames were first bias-subtracted, and cosmic ray hits were detected and removed using the method developed by van Dokkum (2001). The orders were extracted and rectified in wavelength space using a wavelength solution previously obtained from calibration frames. The resulting rectified orders were shifted and coadded to obtain the final two-dimensional spectrum. In the overlapping regions, orders were merged by weighting them according to the errors propagated during the entire reduction process. From the resulting two-dimensional merged spectrum, a onedimensional spectrum was extracted at the source position. The one-dimensional spectrum with the corresponding error file and bad pixel map is the final product of the reduction.

The resolution is $R \sim 10000$ and the achieved spectral range is $\sim 3000$ to $\sim 24800 \AA$. The data below $\sim 3020$ and above $\sim 24000 \AA$ are, however, totally dominated by noise. To perform flux calibration we extracted a spectrum from a staring observation of the flux standard BD $+17 \mathrm{deg} 4708$ (Bohlin \& Gilliland 2004b). In this case we subtracted the sky emission lines using the Kelson (2003) method. This spectrum was divided by the flux table of the same star from the CALSPEC HST database (Bohlin 2007, http://www.stsci.edu/hst/observatory/ cdbs/calspec.html) to produce the response function. The response was then interpolated where needed in the atmospheric absorption bands in VIS and NIR and applied to the spectrum of the source. No telluric correction was applied, so that prominent atmospheric bands, especially in the NIR arm, can still be seen (Fig. 1). We searched for variability in the absorption features, but we found none (the equivalent widths in the four spectra are constant at the $1.5 \sigma$ level), so we summed the four fluxcalibrated observations (see Fig. 1). This lack of variability is not surprising, since the spectra were acquired nearly 1 day after the burst (see Sect. 4.3 for details). The signal-to noise ratio (per pixel) ranges from $\sim 10$ to $\sim 30$ in the co-added spectrum. To perform line fitting (see Sect. 4) the spectrum was normalized to the continuum, which was evaluated by fitting the data with cubic splines, after removing the absorption features. Finally, the noise spectrum, used to determine the errors on the best fit line parameters, was calculated from the real, background-subtracted spectrum using line-free regions. This takes both statistical and systematic errors into account in the pipeline processing and background subtraction.

\section{The host galaxy atomic absorption features}

The gas residing in the GRB host galaxy is responsible for many of the features observed in the GRB 090926A afterglow spectrum. Metallic features are apparent from neutral (O I, Mg I, Ca I), low-ionization (C II, Mg II, Al II, Al III, Si II, S II, Ca II, Fe II, Ni II), and high-ionization (C IV, N V, O VI, Si IV, S IV) species. In addition, strong absorption from the fine structure levels of C II, O I, Si II, Fe II and from the metastable level of $\mathrm{Ni}$ II is identified, suggesting that the intense radiation field from the GRB excites such features. Table 2 gives a summary of all the absorption lines due to the host galaxy gas. The spectral features were analyzed with FITLYMAN (Fontana \& Ballester 1995). This program is able to simultaneously fit several absorption lines, linking the redshifts, column densities and Doppler parameters if required. FITLYMAN takes the atomic masses into account when a thermal model of the Doppler broadening is adopted (as we did), enabling a link to the Doppler parameters of different species. The probed ISM of the host galaxy is resolved into two components separated by $48 \mathrm{~km} \mathrm{~s}^{-1}$, which contribute to the absorption system. The wealth of metal-line transitions allows us to precisely determine the redshift of the GRB host galaxy. This yields a vacuum-heliocentric value of $z=2.1071 \pm 0.0001$, setting the reference point to the red component of the main system; this component is the only one for which there is significant absorption from all the excited levels of the intervening gas (see Sects. 4.1 and 4.3). This redshift value supersedes the one reported by Malesani et al. (2009), which is based on archival calibration data and using an older X-shooter pipeline version. The host-galaxy environment will be described in the next sections, together with a study of the excited lines aimed at estimating the distance from the GRB of the circumburst gas.

\subsection{Line-fitting procedure}

In general, the analysis of the GRB environment is not straightforward owing to the complexity of the absorption line profile, which in several cases cannot be fitted with a single line profile. This means that many components contribute to the gas in the GRB environment. In other words, several layers of gas, whether close to or far from one another, appear mixed together in the spectrum (in velocity space). The presence of several components thus indicates clumpy gas in the GRB environment, composed of different absorbing regions each with different physical properties. This behavior is particularly evident for GRB afterglows observed at high resolution (see e.g. Fiore et al. 2005; Thöne et al. 2008).

For what concerns GRB 090926A, the CIV and Si IV lines have the wider velocity range. This behavior is common to many 
Table 2. Absorption line column densities for the two components of the main system.

\begin{tabular}{|c|c|c|c|}
\hline Species & Observed transitions & $\mathrm{I}\left(0 \mathrm{~km} \mathrm{~s}^{-1}\right)$ & II $\left(-48 \mathrm{~km} \mathrm{~s}^{-1}\right)$ \\
\hline $\mathrm{HI}^{2} \mathrm{~S}_{1 / 2}$ & $\operatorname{Ly} \alpha, \operatorname{Ly} \beta$ & $21.60 \pm 0.07$ & - \\
\hline $\mathrm{C}$ II ${ }^{2} \mathrm{P}_{1 / 2}^{0}$ & $\lambda 1036, \lambda 1334$ & $14.48 \pm 0.04$ & $14.04 \pm 0.04$ \\
\hline $\mathrm{C}_{\mathrm{II}}{ }^{2} \mathrm{P}_{3 / 2}^{0}$ & $\lambda 1037, \lambda 1335$ & $>14.45 \pm 0.04$ & $14.01 \pm 0.05$ \\
\hline $\mathrm{C}$ IV ${ }^{2} \mathrm{~S}_{1 / 2}^{3 / 2}$ & $\lambda 1548, \lambda 1550$ & $14.43 \pm 0.02$ & $13.89 \pm 0.03$ \\
\hline $\mathrm{N} \mathrm{V}{ }^{2} \mathrm{~S}_{1 / 2}$ & $\lambda 1238, \lambda 1242$ & $14.08 \pm 0.03$ & $13.66 \pm 0.07$ \\
\hline $\mathrm{OI}{ }^{3} \mathrm{P}_{2}$ & $\lambda 1039, \lambda 1302$ & $>14.75 \pm 0.03$ & $<13.8$ \\
\hline $\mathrm{OI}^{3} \mathrm{P}_{1}$ & $\lambda 1304$ & $14.49 \pm 0.03$ & $<13.7$ \\
\hline $\mathrm{O}{ }^{3} \mathrm{P}_{0}$ & $\lambda 1306$ & $14.37 \pm 0.04$ & $<13.7$ \\
\hline $\mathrm{OVI}{ }^{2} \mathrm{~S}_{1 / 2}$ & $\lambda 1031, \lambda 1037$ & $>14.60 \pm 0.16$ & Blend \\
\hline $\operatorname{Mg}{ }^{1}{ }^{1} S_{0}$ & $\lambda 2852$ & $12.74 \pm 0.01$ & $11.98 \pm 0.05$ \\
\hline $\mathrm{Mg}$ II $^{2} \mathrm{~S}_{1 / 2}$ & $\lambda 1239, \lambda 1240$ & $>14.05 \pm 0.01$ & $13.05 \pm 0.02$ \\
\hline $\mathrm{Al} \mathrm{II}{ }^{1} \mathrm{~S}_{0}$ & $\begin{array}{c}\lambda 2 / 90, \lambda 2803 \\
\lambda 1670\end{array}$ & $13.20 \pm 0.03$ & $12.66 \pm 0.04$ \\
\hline $\mathrm{Al} \mathrm{III}{ }^{2} \mathrm{~S}_{1 / 2}$ & $\lambda 1854, \lambda 1862$ & $13.24 \pm 0.03$ & $<12.3$ \\
\hline \multirow[t]{4}{*}{$\mathrm{Si}$ II ${ }^{2} \mathrm{P}_{1 / 2}^{0}$} & $\lambda 1020, \lambda 1190$ & $14.41 \pm 0.03$ & $13.98 \pm 0.07$ \\
\hline & $\lambda 1193, \lambda 1260$ & & \\
\hline & $\lambda 1304, \lambda 1526$ & & \\
\hline & $\lambda 1808$ & & \\
\hline \multirow[t]{3}{*}{$\mathrm{Si} \mathrm{II}{ }^{2} \mathrm{P}_{3 / 2}^{0}$} & $\lambda 1194, \lambda 1197$ & $13.96 \pm 0.03$ & $13.42 \pm 0.09$ \\
\hline & $\lambda 1264, \lambda 1309$ & & \\
\hline & $\lambda 1533, \lambda 1309$ & & \\
\hline $\mathrm{Si} \mathrm{III}{ }^{1} \mathrm{~S}_{0}$ & $\lambda 1206$ & $13.50 \pm 0.11$ & Blend \\
\hline $\operatorname{Si~IV~}^{2} \mathrm{~S}_{1 / 2}$ & $\lambda 1393, \lambda 1402$ & $13.97 \pm 0.03$ & $13.61 \pm 0.04$ \\
\hline $\mathrm{S}$ II ${ }^{4} S_{3 / 2}^{0}$ & $\begin{array}{c}\lambda 1250, \lambda 1253 \\
\lambda 1259\end{array}$ & $14.89 \pm 0.06$ & $<14.3$ \\
\hline $\mathrm{S} \mathrm{IV}^{4} \mathrm{~S}_{3 / 2}^{0}$ & $\lambda 1062, \lambda 1253$ & $14.45 \pm 0.19$ & $<14.2$ \\
\hline $\mathrm{Ca} I{ }^{1} \mathrm{~S}_{0}$ & $\lambda 4227$ & $12.15 \pm 0.07$ & $<11.9$ \\
\hline $\mathrm{Ca}$ II ${ }^{2} \mathrm{~S}_{1 / 2}$ & $\lambda 3969$ & $13.21 \pm 0.03$ & $<12.8$ \\
\hline Mn II $a^{7} S_{3}$ & $\lambda 2576$ & $<12.3$ & $<12.3$ \\
\hline \multirow[t]{5}{*}{ Fe II $a^{6} \mathrm{D}_{9 / 2}$} & $\lambda 1081, \lambda 1096$ & $14.03 \pm 0.03$ & $13.46 \pm 0.04$ \\
\hline & $\lambda 1144, \lambda 2344$ & & \\
\hline & $\lambda 2348, \lambda 2374$ & & \\
\hline & $\lambda 2382, \lambda 2586$ & & \\
\hline & $\lambda 2600$ & & \\
\hline \multirow[t]{2}{*}{ Fe II $a^{6} \mathrm{D}_{7 / 2}$} & $\lambda 2333, \lambda 2396$ & $12.52 \pm 0.08$ & $<12.2$ \\
\hline & $\lambda 2612, \lambda 2626$ & & \\
\hline $\mathrm{Fe}$ II $a^{4} \mathrm{~F}_{9 / 2}$ & $\lambda 1559, \lambda 2332$ & $<13.6$ & $<13.6$ \\
\hline $\mathrm{Fe}$ III ${ }^{5} \mathrm{D}_{4}$ & $\lambda 1122$ & $14.54 \pm 0.07$ & $14.38 \pm 0.10$ \\
\hline \multirow[t]{4}{*}{$\mathrm{Ni}$ II ${ }^{2} \mathrm{D}_{5 / 2}$} & $\lambda 1317, \lambda 1370$ & $13.51 \pm 0.06$ & $13.09 \pm 0.30$ \\
\hline & $\lambda 1454, \lambda 1703$ & & \\
\hline & $\lambda 1709, \lambda 1741$ & & \\
\hline & $\lambda 1751$ & & \\
\hline $\mathrm{Ni}$ II ${ }^{4} \mathrm{~F}_{9 / 2}$ & $\begin{array}{c}\lambda 2166, \lambda 2217 \\
\lambda 2316\end{array}$ & $13.54 \pm 0.02$ & $12.47 \pm 0.36$ \\
\hline
\end{tabular}

Notes. All values of the column densities are logarithmic $\left(\right.$ in $\mathrm{cm}^{-2}$ ). Reported lower limits comes from possible line saturation.

GRBs (see e.g. D'Elia et al. 2007; Piranomonte et al. 2008), where these lines are the ones that are most clearly detected so used to guide the identification of the different components constituting the circumburst matter. A two-component model provides a good fit for the C IV and Si IV lines (Fig. 2). Thus, the redshifts and the Doppler parameters of these components were fixed in order to fit the other species present in the spectrum with the same model. This modeling adequately fits all other absorption lines at the GRB redshift. All species feature absorption in the red component, and most of them have absorption also in the blue component. Figures 3 and 4 show the two-component model fit to all the absorption lines at the GRB redshift. Asterisks mark the fine structure levels. The lines Ni II $\lambda \lambda 2166,2217$, and 2316 (Fig. 3, left) are produced by the second excited level of

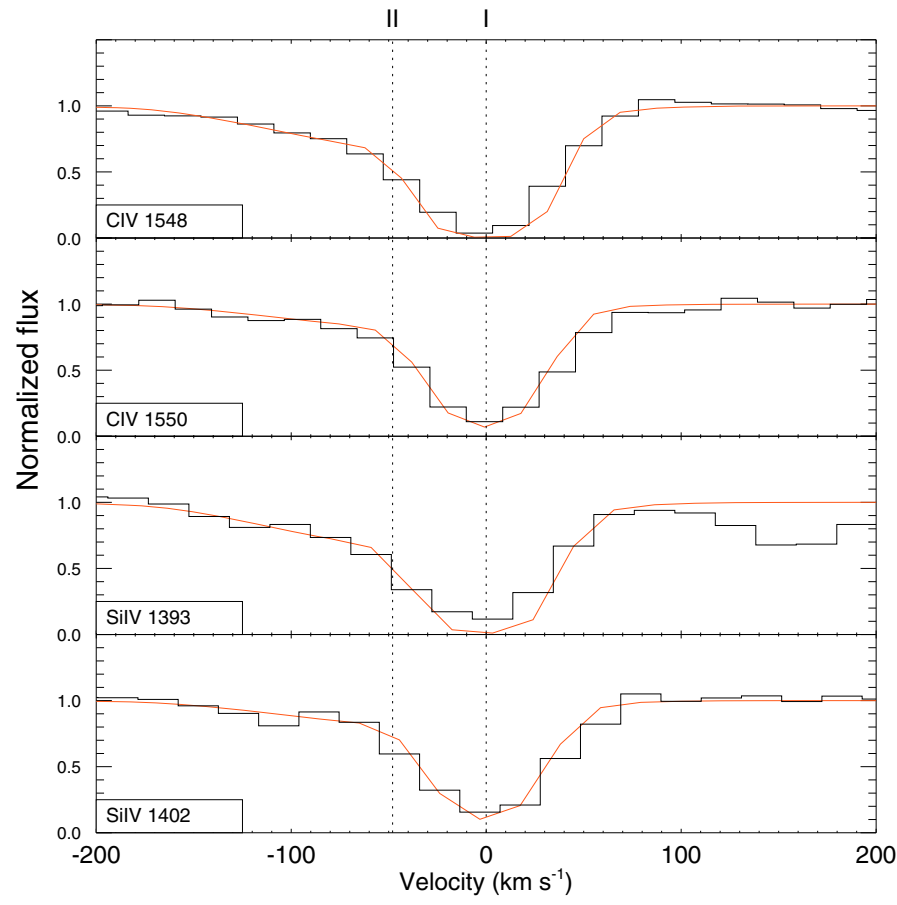

Fig. 2. The C IV and SiIV absorption features. Solid lines represent the two Voigt components, best-fit model. Vertical lines identify the component velocities. The zero point has been arbitrarily placed at the redshift of the red component $(z=2.1071)$.

Ni II $\left({ }^{4} F_{9 / 2}\right)$. The Si III blue component could not be derived because of blending with Ly $\alpha$ forest features (Fig. 4, right).

The column densities for all the elements and ions of the host galaxy's absorbing gas, estimated with this two-component model, are reported in Table 2. The reported upper limits are at the $90 \%$ confidence level. The red and blue components are marked as I and II, respectively. The reference zero point of the velocity shifts has been placed at $z=2.1071$, coincident with the redshift of the red component. Component I of Al III needs a slight wavelength redshift to be adequately fitted (Fig. 3, bottom left). Either way, the resulting column density of this component is consistent with the value obtained by linking the Al III and $\mathrm{C}$ IV central wavelengths. The features of some species, such as Mg II, O I, O VI, and C II, could be saturated at least in component I. In this case, the column densities in Table 2 should be regarded as lower limits to the real values. The column density of Si II has been calculated using only the $\lambda 1304$ transition (see Sect. 4.3). It is interesting to note that among the excited absorption features in the spectrum, produced by fine structure and/or metastable levels, C II, Si II, and Ni II show significant absorption in both components I and II (the absorption in component II from Ni II is marginal), while O I and Fe II only show this in the red, reference component I. We fixed the zero point to the redshift of component I because the Fe II fine structure levels require a high UV flux in order to remain populated (see Sect. 4.3), implying that component I is possibly closer to the GRB explosion site than II. As described in the introduction, the observation of excited states in GRB absorption spectra is quite a common feature. Section 4.3 is thus devoted to these features and to the information that can be extracted from their analysis.

\subsection{Metallicities}

The GRB 090926A redshift was high enough to allow the hydrogen $\operatorname{Ly} \alpha$ and $\operatorname{Ly} \beta$ lines to enter the $\mathrm{X}$-shooter spectral window. 

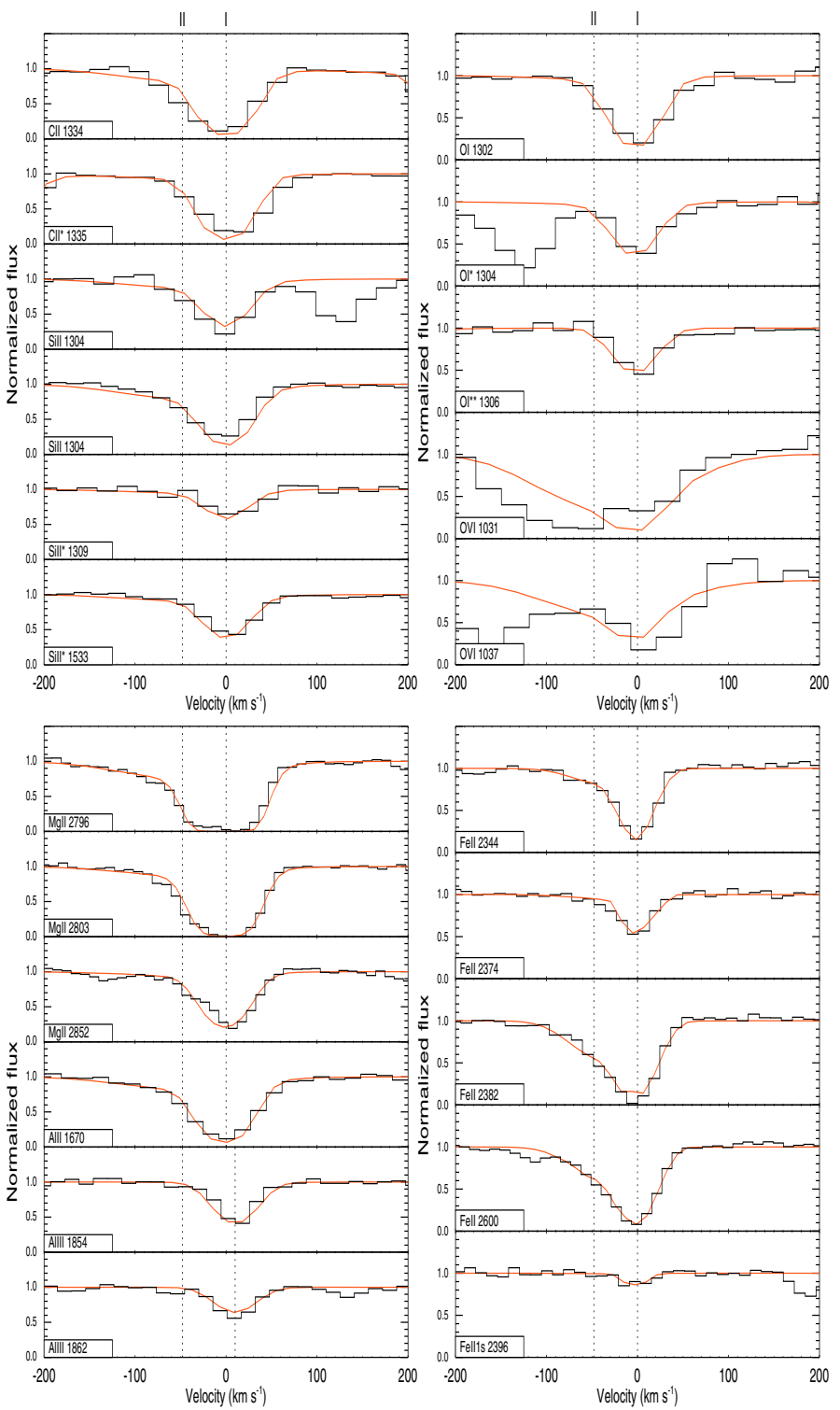

Fig. 3. The C II and Si II (top left panel), O I and O VI (top right panel), Mg I, Mg II, Al II and Al III (bottom left panel), Fe II (bottom right panel) absorption features. Solid lines represent the two Voigt components, best-fit model. Vertical lines identify the component velocities. The zero point has been arbitrarily placed at the redshift of the red component $(z=2.1071)$. The O VI features are blended at blue wavelengths. Component I of $\mathrm{Al}$ III has been slightly shifted for an adequately fit.

Ly $\alpha$ can be clearly seen in Fig. 1 (upper panel) at $\sim 4000 \AA$. We used the two Lyman features to constrain the hydrogen column density. Figure 5 shows the Lyman- $\alpha$ and Lyman- $\beta$ profiles. Both features are damped and we cannot separate the two components identified in the metal-line fits. The hydrogen column density computed has $\log \left(N_{\mathrm{H}} / \mathrm{cm}^{-2}\right)=21.60 \pm 0.07 \mathrm{~cm}^{-2}$, for a reduced $\chi^{2}$ value of 1.33 , and is virtually insensitive to the adopted b parameter. From this $N_{\mathrm{H}}$ value, we can compute the metallicity for the host of GRB 090926A, using the metallic column densities reported in Table 2. The wide spectral coverage of $\mathrm{X}$-shooter allows this to be done for several elements. We proceeded as follows. First of all, since the Lyman features cannot be resolved into components, we summed up line by line the values in Table 2 (e.g., the two components) to obtain the total column density for each species; second, we summed the total column densities of the transitions belonging to the same atom
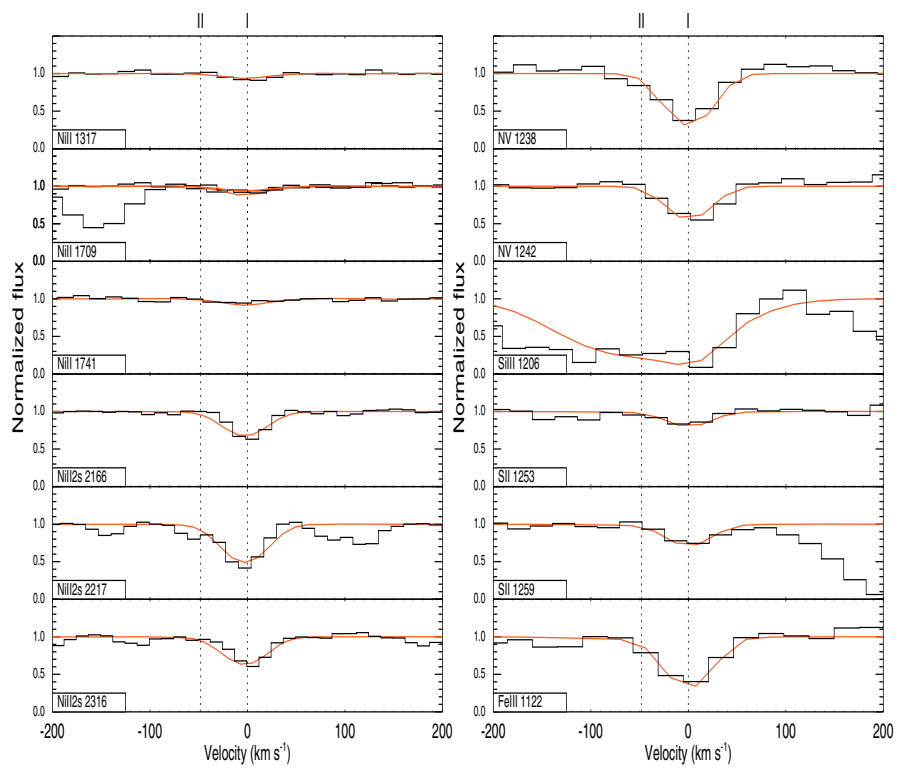

Fig. 4. Left panel: the Ni II absorption features. Right panel: the N V, Si III, S II and Fe III absorption features. Solid lines represent the two Voigt components, best-fit model. Vertical lines identify the component velocities. The zero point has been arbitrarily placed at the redshift of the red component $(z=2.1071)$. The blue component of Si III is blended with absorption from the $\mathrm{Ly} \alpha$ forest.

(different ionization and excitation states), in order to evaluate the atomic column densities. These values were divided by $N_{\mathrm{H}}$ and compared to the corresponding solar values given in Asplund et al. (2009). The results are listed in Table 3. Column 2 reports the total abundance of each atom, while Cols. 3 and 4 report the absolute and solar-scaled $N_{X} / N_{\mathrm{H}}$ ratios, respectively, with $X$ the corresponding element in Col. 1. Lower limits are reported whenever saturation does not allow us to securely fit the metallic column densities. We derived very low metallicity values with respect to the solar ones, between $4.2 \times 10^{-3}$ and $1.4 \times 10^{-2}$. The very low value derived for $\mathrm{N}$ is because we could not fit low-ionization lines, but only the $\mathrm{N} v$ species, meaning that this value is not truly representative of all the $\mathrm{N}$ ionization states.

\subsection{Excited levels}

The level structure of an atom or ion is characterized by a principal quantum number $n$, which defines the atomic level, and by the spin-orbit coupling (described by the quantum number $j$ ), which splits these levels into fine structure sublevels. In GRB absorption spectra, several excited features are detected at the GRB redshift, due to the population of both $n>1$ and/or $n=1$ fine structure levels. As mentioned before, component I of the main system in the spectrum of GRB 090926A shows several absorptions from excited states. In particular, the first fine structure level of the Fe II ground state $\left(a^{6} \mathrm{D}\right)$, the $\mathrm{Si}$ II ${ }^{2} \mathrm{P}_{3 / 2}^{0}$, C II ${ }^{2} \mathrm{P}_{3 / 2}^{0}$, O I ${ }^{3} \mathrm{P}_{1}$, and $\mathrm{O} \mathrm{I}{ }^{3} \mathrm{P}_{0}$ fine structure levels and the $\mathrm{Ni}$ II ${ }^{4} \mathrm{~F}_{9 / 2}$ metastable level are present. Moreover, excited states of Si II, $\mathrm{C}$ II, and (marginally) Ni II are also detected in component II (see Table 2, for details).

There are basically two mechanisms to excite the gas of the GRB's host galaxy to such states. The first is through collisional effects (if the electron density is sufficiently high, i.e., $\geq 10^{5} \mathrm{~cm}^{-3}$ ); the second is through the absorption of electromagnetic radiation. In the latter case, the absorbed photons can be 

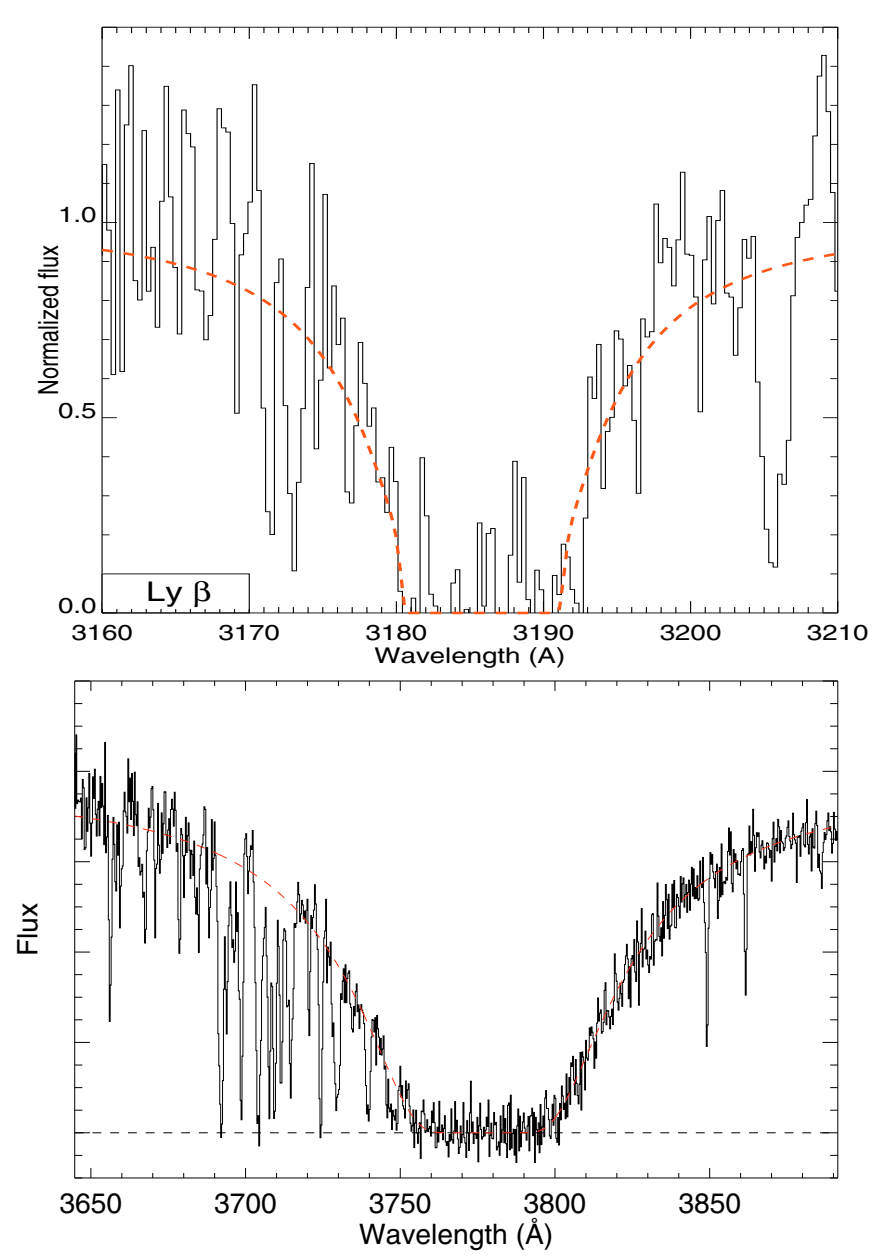

Fig. 5. The Ly $\beta$ (top panel) and Ly $\alpha$ (bottom panel) absorption features at the GRB 090926A redshift. The dashed lines represent the single Voigt component, best-fit model, centered on the redshift of the red component $(z=2.1071)$ of the metallic lines.

infrared, through direct population of the fine structure levels of the ground state and other excited levels with a low value of $n$, or UV, through the population of higher levels followed by the depopulation into the states responsible for the absorption features. Multi-epoch spectroscopy together with proper modeling of the atomic level population has proven to be a powerful tool for distinguishing between these two processes. The strong variability in the column density of the Fe II and Ni II excited levels observed in GRB 060418 (Vreeswijk et al. 2007) and GRB 080319B (D'Elia et al. 2009a) ruled out collisional processes and direct infrared pumping as responsible for the excitation. A collisional origin of the excitation can be ruled out even if multi-epoch spectroscopic data are missing. In fact, collisions populate higher energy levels less than lower energy ones. For instance, GRB 080330 (D'Elia et al. 2009b) and GRB 050730 (Ledoux et al. 2009) exhibit an Fe II $a^{4} \mathrm{~F}_{9 / 2}$ excited state column density that is higher than several fine structure levels of the Fe II $a^{6} D$ ground state. This means that radiative processes are also at work for these GRBs. For GRB 090926A we do have multi-epoch spectroscopy, but the time lag between different observations (a few minutes, see Table 1) is much shorter than the time delay between the GRB explosion and the epoch at which our data were taken. Supported by these results on atomic excitation mechanisms, we assume that indirect UV pumping by the fading afterglow is also at work for GRB 090926A.
Table 3. Metallicities.

\begin{tabular}{lccc}
\hline \hline Element $X$ & $\log N_{X} / \mathrm{cm}^{-2}$ & $\log N_{X} / N_{\mathrm{H}}$ & {$[X / \mathrm{H}]$} \\
\hline $\mathrm{C}$ & $>15.05 \pm 0.10$ & $>-6.55 \pm 0.10$ & $>-2.94 \pm 0.10$ \\
$\mathrm{~N}$ & $14.22 \pm 0.08$ & $-7.38 \pm 0.08$ & $-3.16 \pm 0.08$ \\
$\mathrm{O}$ & $>15.31 \pm 0.10$ & $>-6.29 \pm 0.10$ & $>-2.95 \pm 0.10$ \\
$\mathrm{Mg}$ & $>14.11 \pm 0.08$ & $>-7.49 \pm 0.08$ & $>-3.02 \pm 0.08$ \\
$\mathrm{Al}$ & $13.59 \pm 0.10$ & $-8.01 \pm 0.10$ & $-2.38 \pm 0.10$ \\
$\mathrm{Si}$ & $14.80 \pm 0.08$ & $-6.80 \pm 0.08$ & $-2.31 \pm 0.08$ \\
$\mathrm{~S}$ & $14.89 \pm 0.10$ & $-6.71 \pm 0.10$ & $-1.85 \pm 0.10$ \\
$\mathrm{Ca}$ & $13.25 \pm 0.08$ & $-8.35 \pm 0.08$ & $-2.66 \pm 0.08$ \\
$\mathrm{Fe}$ & $14.86 \pm 0.09$ & $-6.74 \pm 0.09$ & $-2.19 \pm 0.09$ \\
$\mathrm{Ni}$ & $13.92 \pm 0.13$ & $-7.68 \pm 0.13$ & $-1.91 \pm 0.13$ \\
\hline
\end{tabular}

As a first step, we assume a steady state flux coming from the GRB, which in general is not a good approximation. Nevertheless, the GRB 090926A afterglow was observed by X-shooter almost one day after the GRB explosion, and its flux level was decaying slowly at this stage. Under this assumption, we compute the ratio between the first excited level and the ground state for Si II, O I, and Fe II. We then use these values as input for the plot in Fig. 7 of Prochaska et al. (2006) to estimate the flux experienced by the absorbing gas. This plot was produced using the model from Silva \& Viegas (2002), which further assumes optically thin clouds. We first note that, for component I, the Fe II and Si II ratios are not compatible; i.e., the flux levels inferred from these ions are not consistent. This is possily due to a slight saturation of the Si II $\lambda 1526$ line, which was formerly used with the $\lambda 1304$ transition to evaluate the Si II column density. Therefore, we recomputed the Si II column density using just the Si II $\lambda 1304$ line, as described in Sect. 4.1. The flux levels with the new $N_{\mathrm{Si} \text { II }}$ for component I are now fully compatible. We further note that the O I ratio is far from being compatible with that of Si II and Fe II. This confirms the saturation of the O I $\lambda 1302$ transition (Fig. 3, top right), which can just be used to set a lower limit to the O I ground state column density. The Si II ratio for component II is close to the one for I. Thus, we derive a distance of the gas responsible for the absorption of the two components I and II of $d=1.8 \pm 0.2 \mathrm{kpc}$.

To check the results from the steady state approximation, we must compare our observed column densities to those predicted by a photo-excitation code for the time when the spectroscopic observations were acquired. The photo-excitation code is that used by Vreeswijk et al. (2007) and D'Elia et al. (2009a), to which we refer the reader for more details. Basically, it solves the detailed balance equation in a time-dependent way for a set of transitions involving the levels of a given species (e.g. Fe II and Si II). The equation depends on the flux level experienced by the absorbing gas. This flux is, of course, a function of distance $d$ of the gas from the GRB explosion site, which is a free parameter of the computation and the quantity we want to calculate. The other free parameters are the initial column densities of the levels involved, which are assumed to be in the ground state before the GRB flux reaches the gas, and the Doppler parameter of the gas itself. The afterglow spectral index was calculated directly from the merged, flux-calibrated X-shooter spectrum, and is $\beta \sim 0.9$ (see Sect. 6). The flux behavior before the $\mathrm{X}$-shooter observation was estimated using the data in R10. In detail, if the flux in the $R$ band is $F_{R}=F_{R}\left(t_{*}\right) \times\left(t / t_{*}\right)^{-\alpha}$, we have $F_{R}\left(t_{*}\right)=1.1 \times 10^{-27} \mathrm{erg} \mathrm{cm}^{-2} \mathrm{~s}^{-1} \mathrm{~Hz}^{-1}$ at $t_{*}=72.9 \mathrm{ks}$, with a decay index $\alpha$ of $1.6(-2.7)$ before (after) $t_{*}$. No spectral variation is assumed during the time interval between the burst and our observation (an assumption strongly supported by the R10 data). The initial column densities of the ground states were 

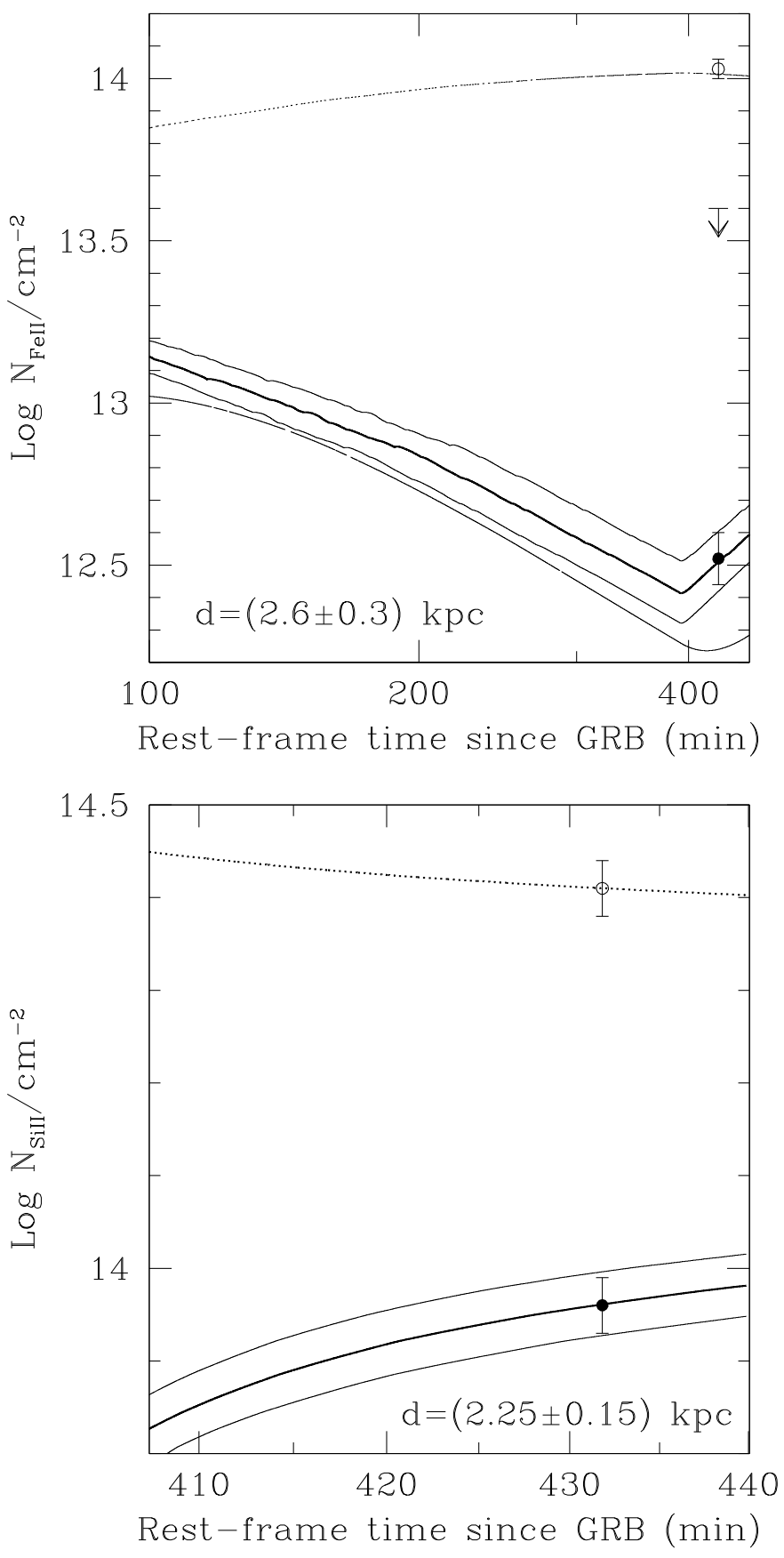

Fig. 6. Top panel: Fe II column densities for the ground level (open circle), first fine structure level (filled circle), and first excited level (upper limit) transitions for component I in the spectrum of GRB 090926A. Column density predictions from our time-dependent photo-excitation code are also shown. They refer to the ground level (dotted line), first fine structure level (thick solid line), and first excited level (dashed line) transitions, in the case of an absorber placed at $2.6 \mathrm{kpc}$ from the GRB. The two thin solid lines display the models that enclose the fine structure data at $1 \sigma$ level. Bottom panel: same as top panel, but for ground and the first fine structure level transitions of Si II.

computed from the observed column densities of all the levels of each ion. The exact values are $\log \left(N_{\mathrm{Si} \text { II }} / \mathrm{cm}^{-2}\right)=14.44 \pm 0.04$ and $\log \left(N_{\mathrm{Fe} \text { II }} / \mathrm{cm}^{-2}\right)=14.04 \pm 0.03$. Finally, the Doppler parameter values used as input of this model are 30 and $90 \mathrm{~km} \mathrm{~s}^{-1}$, i.e. the values that best fit the Fe II and Si II absorptions corresponding to components I and II, respectively.
We first modeled the Fe II photo-excitation. Figure 6 (top) shows the model that best fits the Fe II data and the two theoretical curves compatible within the error bars for the first Fe II fine structure level column density. The distance of component I from the GRB explosion site results $d=2.6 \pm 0.3 \mathrm{kpc}$. The same calculation was performed using the Si II atomic data. The results are displayed in Fig. 6 (bottom), and the estimated distance is $d=2.25 \pm 0.15 \mathrm{kpc}$, which is consistent with what was estimated using the Fe II data. In addition, our distances are less than $30 \%$ away from the estimate with the code by Silva $\&$ Viegas (2002), and compatible with it at the $2 \sigma$ level, confirming that the steady state and optically thin approximations are appropriate for component I of GRB 090926A. Regarding component II, we do not have Fe II excited features, so we can only set a lower limit to the GRB/absorber distance, which is 2.1 (2.2) kpc adopting a Doppler parameter of 30 (90) $\mathrm{km} \mathrm{s}^{-1}$. The Si II fine structure features are instead present in component II. The lack of Fe II excited levels in components featuring Si II fine structure ones is not atypical, see e.g. component III of GRB 050730 identified in D'Elia et al. (2007). Nevertheless, the large errors for this feature and the strong dependence of the model from the Doppler parameter make the distance estimation for this component quite uncertain. We run our code using the Doppler parameters that best-fit components I $\left(30 \mathrm{~km} \mathrm{~s}^{-1}\right)$ and II $\left(90 \mathrm{~km} \mathrm{~s}^{-1}\right)$. The distance of component II from the GRB is found to be $4.4 \pm 0.6 \mathrm{kpc}$ for $b=30 \mathrm{~km} \mathrm{~s}^{-1}$, and $5.8 \pm 0.8 \mathrm{kpc}$ for $b=90 \mathrm{~km} \mathrm{~s}^{-1}$. Despite these values are consistent with the lower limits estimated through Fe II data, we caution that a direct comparison to check the Si II distance is missing.

\section{Other features at the host redshift}

In this section we turn our attention to the search for galactic emission lines (Sect. 5.1) and absorption features not caused by matter in the atomic gas phase. The latter can be produced by molecules (Sect. 5.2) or DIBs (Sect. 5.3).

\subsection{Emission lines from the host galaxy}

We searched for most of the stronger emission lines like [O II] $\lambda$ 3727, [O III] $\lambda$ 5007, and the Balmer lines from the host galaxy in the X-shooter spectrum, but found none. We did this by subtracting the spectral PSF. To derive upper limits for say $\mathrm{H} \alpha$, we then added artificial emission lines of increasing strength to the data, until the line was easily detected. For $\mathrm{H} \alpha$, which in this case is observed at $2 \mu \mathrm{m}$, we find that an emission line of $9 \times 10^{-18} \mathrm{erg} \mathrm{s}^{-1} \mathrm{~cm}^{-2}$ with an intrinsic velocity width of $100 \mathrm{~km} \mathrm{~s}^{-1}$ would have been detected (barring slit losses). This means that we would have detected emission lines from a host galaxy with a star formation rate $\gtrsim 2 M_{\odot} \mathrm{yr}^{-1}$ (Kennicutt 1998). We also note that there is no $\operatorname{Ly} \alpha$ emission in the trough of the DLA. The $5 \sigma$ detection limit in a $6 \AA$ wide extraction window is $3.0 \times 10^{-17} \mathrm{erg} \mathrm{s}^{-1} \mathrm{~cm}^{-2}$ corresponding to $S F R<1 M_{\odot} \mathrm{yr}^{-1}$. However, this limit is much more senstitive to uncertainties in the radiative transfer of Ly $\alpha$ photons and to host galaxy dust extinction (see, e.g., Hayes et al. 2010). Although there is no evidence of dust extinction from the SED of the afterglow radiative transfer can still completely remove the Ly $\alpha$ emission from the line-of-sight (e.g., Atek et al. 2008). Many GRB host galaxies do have higher SFR than this limit (e.g., Christensen et al. 2004), so we will be able to detect emission lines from many high- $z$ GRB hosts using X-shooter in the future. 


\subsection{Molecular absorption features}

Molecular gas is expected to be found in star-forming environments, but the search for its absorption features has often given negative results (see e.g. Tumlinson et al. 2007). Fynbo et al. (2006) interpreted an absorption feature of the GRB 060206 afterglow spectrum as a possible $\mathrm{H}_{2}$ detection. Prochaska et al. (2009) and Sheffer et al. (2009) report the presence of strong $\mathrm{H}_{2}$ and $\mathrm{CO}$ absorption features in the spectrum of the GRB 080607 afterglow. These are the first (and by now unique) positive detections of molecules in GRB host galaxies. For GRB 090926A, we searched for the strongest features of the $\mathrm{CO}$ molecule, e.g., CO $\lambda \lambda 1510,1478$ and 1447, but we found no absorption at these wavelengths. Quantitatively, the upper limit for the CO column density depends on the adopted Doppler value $b$. Assuming it is in the range $30-90 \mathrm{~km} \mathrm{~s}^{-1}$ (i.e., the values that best fits components I and II, respectively, see Sect. 4.1), the resulting upper limit for the CO column density lies in the interval $\log \left(N_{\mathrm{CO}} / \mathrm{cm}^{-2}\right)<14-14.3$. We also searched for $\mathrm{H}_{2}$ absorption. The strongest $\mathrm{H}_{2}$ transitions of the Lyman-Werner bands have a wavelength $<3000 \AA$ at the redshift of GRB 090926A. Nevertheless, using the $\mathrm{H}_{2}$ L2R0 transition, which falls around $3350 \AA$, we can set a $90 \%$ upper limit of $\log \left(N_{\mathrm{H}_{2}} / \mathrm{cm}^{-2}\right)<14.9$ for a $b$ parameter fixed at $30 \mathrm{~km} \mathrm{~s}^{-1}$ and $\log \left(N_{\mathrm{H}_{2}} / \mathrm{cm}^{-2}\right)<15.3$ for $b$ fixed at $90 \mathrm{~km} \mathrm{~s}^{-1}$. Finally, we searched for other absorptions due to the $\mathrm{CH}, \mathrm{CH}^{+}$, and $\mathrm{CN}$ molecules, but also in these cases we found none.

\subsection{Diffuse interstellar bands}

DIBs (see Jenniskens \& Desert 1994, and references therein) are broad absorption features in the near-infrared to UV wavelength range. They were observed for the first time more than 70 years ago in the Milky Way (Merril 1934) and, more recently, in near galaxies (see e.g., Cox \& Cordiner 2008; Cordiner et al. 2008). Although they have been known for a long time and hundreds of features have already been identified, the nature of the carrier of such transitions is still uncertain. The identification is difficult since most of them are not correlated with each other. The most promising candidates are large molecules, which are thought to be polycyclic aromatic hydrocarbons. We searched for the ten strongest and most common DIBs in the GRB 090926A afterglow spectrum, in the rest frame wavelength range $\lambda=4000-7000 \AA$, but we made no positive detection. A conservative $2 \sigma$ upper limit to the rest frame equivalent width of these systems (considering that they have very different $F W H M)$ is $E W<0.3 \AA$. This limit is set by the signal-to-noise ratio of the infrared arm of our $\mathrm{X}$-shooter spectrum, i.e., is the "noise $E W$ ".

\section{The extinction curve shape}

The shape of extinction curves at high redshift is a powerful tool for deriving information about dust formation and possibly about the various processes affecting dust absorption and destruction close to GRB sites. In most cases, inferences about dust extinction curves are obtained by photometric observations of GRB afterglows, which unfortunately is biased by a strong degeneracy between afterglow spectral slope and extinction. Moreover, for very high redshift events in particular, uncertainties in photometry of single bands, which can also present specific calibration problems may affect the whole analysis (see for instance discussion in Stratta et al. 2007; Zafar et al. 2010 about GRB 050904).
For early time afterglows, even the possible non-absolute simultaneity of the available photometric information has to be properly considered to avoid spurious results.

For most GRB afterglows, when accurate multi-band photometry is available (e.g., Covino et al. 2008; Schady et al. 2010; Kann et al. 2010), the derived extinction curve is in fair agreement with what is observed locally in the Small Magellanic Cloud (SMC, Pei 1992), although often, due to the limited wavelength resolution, this simply means that the observed extinction curve has to be chromatic (i.e. wavelength dependent) and featureless. A few remarkable exceptions have been recorded in an intervening system along the line of sight of GRB 060418 (Vreeswijk et al. 2007), for GRB 070802 (Krühler et al. 2008; Elíasdóttir et al. 2009) and GRB 080607 (Prochaska et al. 2009), where the characteristic absorption feature at about $2175 \AA$, prominent in the Milky Way extinction curve, has also been detected.

Clearly, when spectroscopic information is available (e.g., Liang \& Li 2010), better constrained results can be derived, disentangling the extinction curve and spectral slope effects. The case of GRB 090926A is a good example of the X-shooter capabilities in this context. The flux-calibrated spectrum (Fig. 1) has been analyzed after removing wavelength intervals affected by telluric lines of strong absorptions. The Ly $\alpha$ range has been included in the analysis because its wavelength range is important for extinction determination using the hydrogen column density reported in Table 2 . We then rebin the spectrum in bins of approximately $50 \AA$, by a sigma-clipping algorithm to avoid the effect of residual absorption systems. The flux-calibration of $\mathrm{X}$-shooter spectra ranging from the UV to the $K$ band is not an easy task, and the reduction pipeline is still under active development (Goldoni et al. 2006). To obtain an acceptable fit, we had to introduce additional systematic uncertainties at about $\sim 2.5 \%$ level added in quadrature, which possibly reflects normalization biases of the three arms and/or still not fully modeled slit losses in the whole wavelength range. Assuming a power-law spectrum, we obtained $\beta=0.89 \pm 0.02$ (errors at $1 \sigma$ ) where the afterglow spectrum is modeled, as is customary in GRB literature, as $F_{v} \propto v^{-\beta}$. We tried to fit the data using different extinction curves, namely, SMC, LMC, Milky Way, and Starburst. The best fit is obtained assuming an SMC extinction curve with $E_{B-V}<0.01 \mathrm{mag}$ at $3 \sigma\left(\chi^{2} /\right.$ d.o.f. $=1.14$ for 484 d.o.f. $)$. Other extinction curve recipes were not required by the data.

\section{The GRB 090926A line of sight}

The absorption lines due to the gas belonging to the GRB host galaxy are the dominant features of our spectrum, but they are not the only ones. A detailed analysis of the data reveals that at least four other absorbers are present along the line of sight to GRB 090926A. Three of these systems, those with the highest redshifts $(z=1.75-1.95)$, show absorption from the C IV $\lambda \lambda 1548,1550$ doublet, to which a well-defined H I $\lambda 1215$ line corresponds inside the Ly $\alpha$ forest. All these systems exhibit a very simple line profile that can be fitted by a single Voigt function (Fig. 7). The Ly $\alpha$ of the system at $z=1.7986$ requires a Doppler parameter that is about three times what it is for the C IV doublet to be adequately fit. If we assume a double component model for this Lyman- $\alpha$ feature and fix the $b$ parameter of one component to that of the C IV doublet, the estimated $N_{\mathrm{H}}$ column density is not significantly different from what is computed using the single-component model. The last of the GRB 090926A intervening systems has a lower redshift $(z=1.2456)$ and features absorption from the $\mathrm{Mg}$ II $\lambda \lambda 2796,2803$ doublet, and marginally 

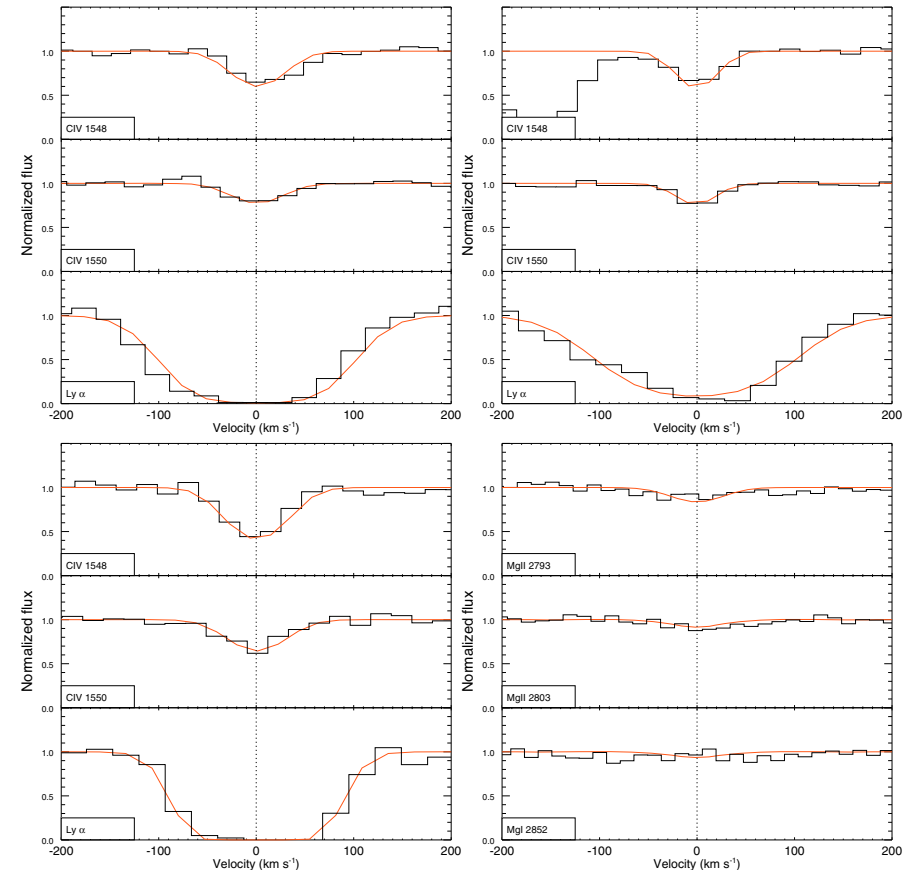

Fig. 7. The four intervening systems along the GRB090926A sightline. Top left: intervening 1 at $z=1.9466$; top right: intervening 2 at $z=1.7986$; bottom left: intervening 3 at $z=1.7483$; bottom right: intervening 4 at $z=1.2456$. The first three systems feature the C IV doublet and Ly $\alpha$. System 4 shows Mg I and Mg II, with the $\lambda 2852$ transition and $\lambda 2796-\lambda 2803$ doublet, respectively. Mg I is marginally detected $(2.7 \sigma)$. Solid lines represent our best-fit model (see also Table 4).

$(2.7 \sigma)$ from the $\operatorname{Mg} \mathrm{I} \lambda 2852$ line. This is a rather weak system, and the rest frame equivalent width for the $\mathrm{Mg}$ II $\lambda 2796$ line is just $E W_{\mathrm{r}}=0.19 \pm 0.06 \AA(2 \sigma$ confidence $)$. Again, a singlecomponent Voigt profile describes the absorption features well (Fig. 7).

Table 4 summarizes the rest frame equivalent widths, column densities, and redshifts calculated for these intervening systems.

\section{Conclusions and discussion}

In this paper we present intermediate-resolution $(R=10,000)$ spectroscopy of the optical afterglow of GRB 090926A, observed using the $\mathrm{X}$-shooter spectrograph at the $V L T \sim 22 \mathrm{~h}$ after the trigger. From the detection of hydrogen and metal absorption features, we find that the heliocentric redshift of the host galaxy is $z=2.1071$. The spectrum shows that the ISM of the GRB host galaxy has at least two components contributing to this main absorption system at $z=2.1071$. Such components, whose line centers are separated by $\sim 48 \mathrm{~km} \mathrm{~s}^{-1}$, are identified in this paper as I and II, according to their decreasing velocity values. The total width of the two components is $\sim 250 \mathrm{~km} \mathrm{~s}^{-1}$. We stress that identifying just two components may be due to the X-shooter resolution. In fact, GRB afterglows observed with higher spectral resolution feature more complex environments either if the host galaxy absorber has a similar width (e.g., 7 components for GRB 050922C, see Piranomonte et al. 2008 or even for smaller widths (GRB 080319B has six components for a total width of $\sim 120 \mathrm{~km} \mathrm{~s}^{-1}$, see D'Elia et al. 2009a).

The absorption lines appear both as neutral metal-absorption, low ionization, and high ionization species. In addition, strong absorption from the fine structure and metastable levels of several species are detected. The distances between the GRB and
Table 4. Redshifts, absorption line column densities, and equivalent widths for the intervening systems.

\begin{tabular}{cccccc}
\hline \hline & Species & Transition & Redshift & $\log \left(N / \mathrm{cm}^{-2}\right)$ & $E W_{\mathrm{r}}(\AA)^{a}$ \\
\hline 1 & CIV & 1548,1550 & 1.9466 & $13.70 \pm 0.03$ & $0.15 \pm 0.04$ \\
1 & HI & Ly $\alpha$ & 1.9466 & $14.64 \pm 0.04$ & \\
2 & CIV & 1548,1550 & 1.7986 & $13.63 \pm 0.03$ & $0.11 \pm 0.03$ \\
2 & HI & Ly $\alpha$ & 1.7986 & $14.56 \pm 0.07$ & \\
3 & CIV & 1548,1550 & 1.7483 & $13.90 \pm 0.02$ & $0.21 \pm 0.03$ \\
3 & HI & Ly $\alpha$ & 1.7483 & $14.98 \pm 0.41$ & \\
4 & MgII & 2796,2803 & 1.2456 & $12.39 \pm 0.05$ & $0.19 \pm 0.06$ \\
4 & MgI & 2852 & 1.2456 & $11.47 \pm 0.13$ & \\
\hline
\end{tabular}

Notes. ${ }^{(a)}$ Rest frame equivalent widths for Mg II $\lambda 2796$ and C IV $\lambda 1548$; $E W$ errors are given at the $2 \sigma$ confidence level.

the two absorbers were estimated using the code by Silva \& Viegas (2002), in a steady state and optically thin approximation, using the ratios between the ground state and the first excited levels of different species to infer the flux level experienced by the absorbing gas. For both components we find that the absorber is located at a distance of $d=1.8 \pm 0.2 \mathrm{kpc}$.

The value of this distance can be refined by comparing the column densities of the ground and excited levels to those predicted by a time-dependent photo-excitation code. Using Fe II and Si II, we find that the absorbing gas of component I is located at $d=2.40 \pm 0.15 \mathrm{kpc}$ from the GRB, a value that is not far from what is estimated when assuming a steady state approximation. For component II, this distance is greater, $\sim 5 \mathrm{kpc}$, but this value has only been obtained using Si II, because we only have a lower limit for the Fe II fine structure column density. Even though this lower limit gives a distance consistent to the Si II result, a safe cross check cannot be performed.

The GRB 090926A/absorber distance is compatible with what is found for the 4 other GRBs for which a similar analysis has been performed, i.e., $1.7 \mathrm{kpc}$ for GRB 060418 (Vreeswijk et al. 2004), 2-6 kpc for GRB 080319B (D'Elia et al. 2009a), 280 pc for GRB 080330 (D'Elia et al. 2009b) and 440 pc for GRB 050730 (Ledoux et al. 2009). This is further confirmation that the power of a GRB affects a region of gas that is at least a few hundreds pc in size.

Several high ionization lines are detected in the GRB090926A spectrum (see Table 2). Our Nv column density is within the range of the GRB sample studied by Prochaska et al. (2008), who claim that this ion can be located very close to the GRB explosion site. Fox et al. (2008) estimated a lower limit to the S IV distance from the absorber using the non detection of the S IV fine structure feature and a photoexcitation code similar to that used in this paper. They conclude that for GRB 050730 this ion is located at distances over 400 pc. An upper limit of the same order of magnitude can also be roughly estimated for GRB090926A, since we compute a tighter limit for the $\mathrm{SIV}^{*}$ column density of $\log \left(N_{\mathrm{S} \text { IV }} / \mathrm{cm}^{-2}\right)<13.3$, but the flux of our GRB is about three times less than that of GRB 050730 (compared at the time of acquiring the spectra). In addition, Fox et al. (2008) report the detection in six of the seven GRB spectra analyzed in their work of CIV, high-velocity components at 500-5000 $\mathrm{km} \mathrm{s}^{-1}$. This gas can belong to foreground clouds but also be associated to the Wolf-Rayet wind of the GRB progenitors. We do not find any such absorptions in the spectra of GRB 090926A.

The redshift of GRB 090926A allows us to determine the hydrogen column density, which has $\log \left(N_{\mathrm{H}} / \mathrm{cm}^{-2}\right)=21.60 \pm 0.07$. 
This value is lower than found by R10 $\left(\log \left(N_{\mathrm{H}} / \mathrm{cm}^{-2}\right)=21.79 \pm\right.$ $0.07)$, but the $2 \sigma$ regions overlap, and each value is consistent with the other at the $2.7 \sigma$ level. Using $N_{\mathrm{H}}$ we evaluate the GRB 090926A host galaxy's metallicity. The values we find are in the range $10^{-3}-10^{-2}$ with respect to the solar abundances (see Table 3). R10 report a metallicity calculated using S II, Si II, Al II, O I, and Fe II. The first three values agree perfectly with our estimates for $\mathrm{S}, \mathrm{Si}$, and $\mathrm{Al}$. Their $\mathrm{OI}$ value is consistent with our oxygen lower limit (set mainly by the saturated O I features). However, the authors note that their extremely low value $(\sim-3.08)$ is possibly caused by saturation, and it could consequently lead to underestimating the O I column density, and thus this should be regarded as a lower limit as well. Another way to derive a metallicity using oxygen is to evaluate the O I ground state column density through the fine structure one, in the hypothesis of a steady state UV pumping as responsible for this excitation. This has proven to be a good approximation for component I, the only one featuring O I. Under this assumption, we obtain $\log \left(N_{\mathrm{O}} / \mathrm{cm}^{-2}\right) \sim 16.05$ and a metallicity of $[\mathrm{O} / \mathrm{H}]=-2.18 \pm 0.16$, which is consistent with the values obtained using other elements. Finally, the R10 Fe II abundance $([\mathrm{Fe} / \mathrm{H}] \sim-2.93)$ is not consistent with our iron value of $[\mathrm{Fe} / \mathrm{H}]$ $=-2.19 \pm 0.07$. This is because we considered the Fe III $\lambda 1122$ line, which brings a relevant contribution to the total iron column density for GRB 090926A. Due to this contribution, we do not detect an overabundance of silicon with respect to iron, contrary to what is reported by $\mathrm{R} 10$.

For our results, the extremely low value derived for nytrogen $([\mathrm{N} / \mathrm{H}] \sim-3.31)$ most certainly comes from our not being able to fit low ionization lines, but only the $\mathrm{N} v$ species. For calcium $([\mathrm{Ca} / \mathrm{H}] \sim-2.71)$, we cannot exclude possible contamination by sky lines. Despite this, we still have metallicity in the range $4.2 \times 10^{-3}-1.4 \times 10^{-2}$ with respect to solar. The average, logarithmic metallicity is $[\mathrm{X} / \mathrm{H}]=-2.14 \pm 0.09$ and is consistent with computations by the R10 data excluding oxygen and iron. This value lies at the lower end of the GRB distribution (Savaglio 2006; Prochaska et al. 2007; Savaglio et al. 2009); in fact, only GRB 050730 and GRB 050922C have metallicity values below $10^{-2}$.

A powerful way to infer the nature and the age of objects whose morphology is unknown is with abundances and abundance ratios (see Matteucci 2001). This method is based on the fact that galaxies of different morphological type are characterized by different star formation histories, and these strongly influence the $[X / \mathrm{Fe}]$ versus $[\mathrm{Fe} / \mathrm{H}]$ behavior. For very high star formation rates, as expected in spheroids (bulges and ellipticals) in their evolution phases, the $[\alpha / \mathrm{Fe}]$ ratios are more overabundant than the Sun in a wide range of $[\mathrm{Fe} / \mathrm{H}]$ values, because in a regime of strong star formation the large number of type II $\mathrm{SNe}$ acting in the early phases of galaxy evolution increases the $[\mathrm{Fe} / \mathrm{H}]$ in the gas up to high values (almost solar and solar) on timescales too short for the type Ia SNe to substantially enrich the gas in iron. Therefore, the $[\alpha / \mathrm{Fe}]$ ratios will reflect the production ratio of SNe II, which is greater than in the solar birthplace. In fact, the bulk of iron is supposed to have been produced by type Ia SNe. On the other hand, objects evolving slowly with a lower star formation rate than normal ellipticals, such as irregular galaxies, would show low $[\alpha / \mathrm{Fe}]$ ratios even at low $[\mathrm{Fe} / \mathrm{H}]$ values. This is because in this case $\mathrm{SNe}$ Ia have time to pollute the gas much before it reaches the Fe solar value. Therefore, if we compare the measured abundance ratios in the host of GRB 090926A with predictions from detailed chemical evolution models, we should be able to understand the nature of the host. This is done in Fig. 8 where models for ellipticals

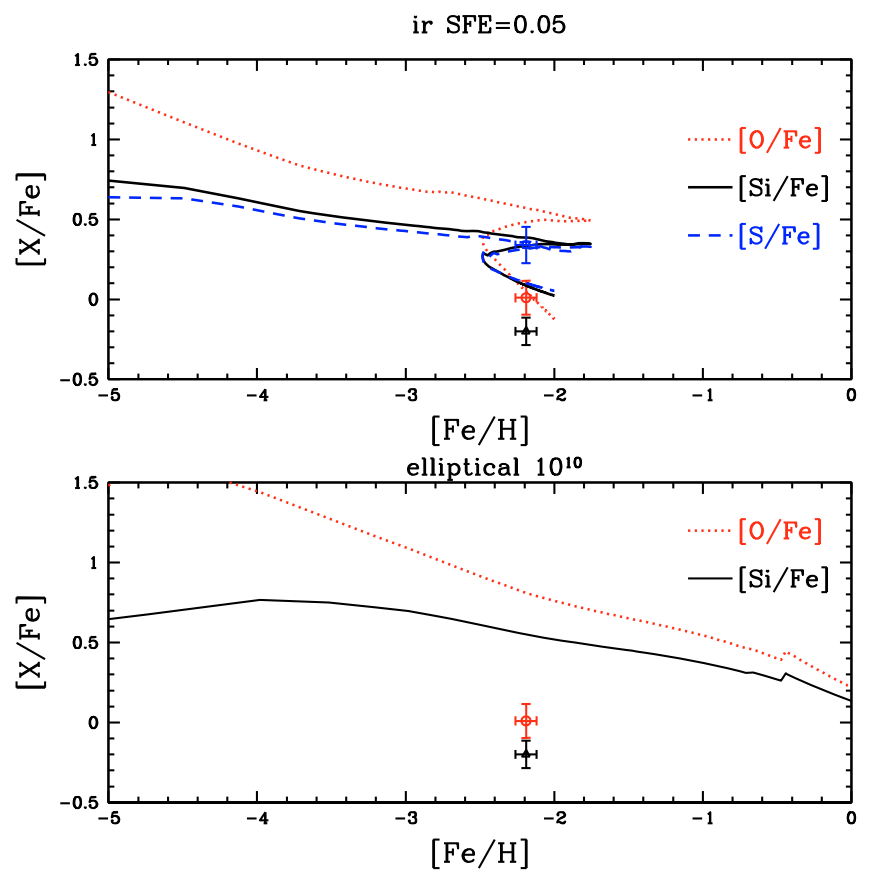

Fig. 8. Predicted $[X / \mathrm{Fe}]$ vs. $[\mathrm{Fe} / \mathrm{H}]$ for a typical irregular galaxy (upper panel) with a baryonic mass of $10^{8} M_{\odot}$ (star formation efficiency of $0.05 \mathrm{Gyr}^{-1}$ ) and for a small elliptical with baryonic mass $M=10^{10} M_{\odot}$ (lower panel). The data for GRB 090926A are shown for comparison.

and for irregular galaxies are compared with the measured abundances. These models, which are discussed in Fan et al. (in prep.), show that the host of GRB 090926A is probably an irregular galaxy with baryonic mass $10^{8} M_{\odot}$ and evolving with star formation efficiency (the inverse of the timescale of star formation) of $0.05 \mathrm{Gyr}^{-1}$. In fact, the measured $[\alpha / \mathrm{Fe}]$ ratios agree better with the prediction for the irregular galaxies and are instead too low for an elliptical galaxy of baryonic mass $10^{10} M_{\odot}$, shown for comparison. The model for the irregular galaxy takes metalenhanced galactic winds into account, induced by SN feedback, which produce the loops in the predicted abundance ratios.

This kind of host galaxy, associated with the low metallicity of the GRB 090926A ISM, agrees with the tentative evidence of a trend to declining ISM metallicity with decreasing galaxy luminosity in the star-forming galaxy population at $z=2-4$ (Chen et al. 2009).

We also searched for other features at the host galaxy redshift. No emission lines were detected for the GRB 090926A host, but our lower limits ensure that we will be able to detect emission lines from many high-redshift GRB hosts using $\mathrm{X}$-shooter in the future. Similarly, we find no evidence of molecular absorption at the GRB redshift. The lower limit on $\mathrm{H}_{2}$ translates into an upper limit for the hydrogen molecular fraction of $f=2 N_{\mathrm{H}_{2}} /\left(2 N_{\mathrm{H}_{2}}+N_{\mathrm{H}}\right)<(3.0-7.4) \times 10^{-7}$. The absence of molecules is not surprising, since hydrogen molecular fractions $\log f>-4.5$ are detected in just $10 \%$ of the QSO-DLA population. In addition, GRB environments with metallicities below $0.1 Z_{\odot}$ (such as that of GRB 090926A) and low dust content can explain the lack of $\mathrm{H}_{2}$ (see Ledoux et al. 2009). As a confirmation of this scenario, the only positive detection of molecules in GRB host galaxies has been reported in the GRB 080607 afterglow, for which a solar or even super-solar metallicity has been inferred (Prochaska et al. 2009). Finally, DIBs have not been detected either.

The GRB 090926A continuum has been fitted assuming a power-law spectrum. The best-fit spectral index is $\beta=0.89_{-0.02}^{+0.02}$ 
$(1 \sigma)$. This value is consistent with the one obtained in R10 by fitting the GROND data $\left(0.98_{-0.07}^{+0.06}\right)$, and is close to that obtained by the same authors fitting the optical/IR and the X-ray data together $(\sim 1.03)$. Our best fit does not essentially allow for any intrinsic extinction since $E_{B-V}<0.01 \mathrm{mag}$ at $3 \sigma$ adopting an SMC extinction curve. Other extinction curve recipes are not required by the data. This intrinsic extinction limit is consistent with what is reported by R10.

The GRB 090926A sightline has also been analyzed. The signal-to-noise level of our spectrum allowed us to be sensitive to lines with equivalent width (observed frame) as weak as $0.06-0.15 \AA$ (depending on the spectral region), at the $2 \sigma$ confidence level. The redshift path analyzed for the search of $\mathrm{Mg}$ II (C IV) systems ranges from $z=2.107$ to $z=0.35(z=1.44)$. Four intervening systems between $z=1.95$ and $z=1.24$ have been identified. All systems have very small rest frame equivalent widths, below $0.3 \AA$. Two of these systems, marked as 1 and 3 in Table 4, have also been reported by R10, and their equivalent widths are consistent with ours. The line of sight toward GRB 090926A is thus very clean, if compared to other GRBs. In fact, Prochter et al. (2006) and Vergani et al. (2009) claimed an excess of strong ( $E W>1 \AA) \mathrm{Mg}$ II absorbers along GRB sight lines with respect to QSO's. On the other hand, the number of $\mathrm{C}$ IV and weak Mg II systems is consistent along the line of sight of the two classes of objects (Tejos et al. 2007, 2009). The reason for the strong Mg II discrepancy is still uncertain, even though several possibilities have been already ruled out (e.g Porciani et al. 2007; D'Elia et al. 2010). The only proposed explanation that is still a possible candidate for this discrepancy is a multiband magnification bias in GRB sightlines (Vergani et al. 2009). More observations and analysis are needed to solve this issue.

Acknowledgements. We thank an anonymous referee for several helpful comments that improved the quality and clarity of the paper. The Dark Cosmology Center is funded by the Danish National Research Foundation.

\section{References}

Asplund, M., Grevesse, N., Sauval, A. J., \& Scott, P. 2009, ARA\&A, 47, 481 Atek, H., Kunth, D., Hayes, M., Östlin, G., \& Mas-Hesse, J. M. 2008, A\&A, 482, 491

Bissaldi, E. 2009, GCN Circ, 9933

Bohlin, R. C. 2004a, in ASP Conf. Ser., 364, 315

Bohlin, R. C., \& Gilliland, R. L. 2004b, AJ, 128, 3053

Chen, H. W., Perley, D. A., Pollack, L. K., et al. 2009, ApJ, 691, 152

Christensen, L., Hjorth, J., \& Gorosabel, J. 2004, A\&A, 425, 913

Cordiner, M. A., Smith, K. T., Cox, N. L. J., et al. 2008, A\&A, 492, L5

Covino, S., D’Avanzo, P., Klotz, A., et al. 2008, MNRAS, 388, 347

Cox, N. L. J., \& Cordiner, M. A. 2008, IAUS, 251, 237

Cucchiara, A., Jones, T., Cherlton, J. C., et al. 2009, ApJ, 693, 345

D’Elia, V., Fiore, F., Perna, R., et al. 2009a, ApJ, 694, 332

D’Elia, V., Fiore, F., Perna, R., et al. 2009b, A\&A, 503, 437
D'Elia, V., Fiore, F., Goldoni, P., et al. 2010, MNRAS, 401, 385

de Ugarte Postigo, A., Goldoni, P., Thöne, C. C., et al. 2010, A\&A, 513, 42

D'Odorico, S., Dekker, H., Mazzoleni, R., et al. 2006, in SPIE Conf. Ser., 6269

Elíasdóttir, Á., Fynbo, J. P. U., Hjorth, J., et al. 2009, ApJ, 697, 1725

Fiore, F., D'Elia, V., Lazzati, D., et al. 2005, ApJ, 624, 853

Fontana, A., \& Ballester, P. 1995, The Messenger, 80, 37

Fox, A. J., Ledoux, C., Vreeswijk, P. M., Smette, A., \& Jaunsen, A. O. 2008, A\&A, 491, 189

Fynbo, J. P. U., Starling, R. L., Ledoux, C., et al. 2006, A\&A, 451, L47

Fynbo, J. P. U., Prochaska, J. X., Sommer-Larsen, J., Dessauges-Zavadsky, M., \& Moller, P. 2008, ApJ, 683, 321

Goldoni, P., Royer, F., Francois, P., et al. 2006, in Society of Photo-Optical Instrumentation Engineers (SPIE), Conf. Ser., 6269

Gronwall, C., \& Vetere, L. 2009, GCN Circ 9938

Haislip, J., Reichart, D., Ivarsen, K., et al. 2009, GCN Circ 9937

Hayes, M., Östlin, G., Schaerer, D., et al. 2010, Nature, 464, 562

Jenniskens, P., \& Desert, F.-X. 1994, A\&AS, 39, 206

Kann, D. A., Klose, S., Zhang, B., et al. 2010, ApJ, 720, 1513

Kelson, D. D. 2003, PASP, 115, 688

Kennicutt, R. C. 1998, ApJ, 498, 541

Krühler, T., Küpcü Yoldaş, A., Greiner, J., et al. 2008, ApJ, 685, 376

Ledoux, C., Vreeswijk, P. M., Smette, A., et al. 2009, A\&A, 506, 661

Liang, S. L., \& Li, A. 2010, ApJ, 710, 1

Malesani, D., Goldoni, P., Fynbo, J. P. U., et al. 2009, GCN Circ 9942

Matteucci, F. 2001, AASL (Kluwer Academic Publisher)

Merril, P. W. 1934, PASP, 46, 206

Oates, S. R. 2010, GCN 9948

Pei, Y. C. 1992, ApJ, 395, 130

Piranomonte, S., Ward, P. A., Fiore, F., et al. 2008, A\&A, 492, 775

Porciani, C., Viel, M., \& Lilly, S. J. 2007, ApJ, 659, 218

Prochaska, J. X., Chen, H. W., \& Bloom, J. S. 2006, ApJ, 648, 95

Prochaska, J. X., Chen, H. W., Dessauges-Zavadsky, E., \& Bloom, J. S. 2007, ApJ, 666, 267

Prochaska, J. X., Dessauges-Zavadsky, M., Ramirez-Ruiz, M., \& Chen, H. W. 2008, ApJ, 685, 344

Prochaska, J. X., Sheffer, Y., Perley, D. A., et al. 2009, ApJ, 691, L27

Prochter, G. E., Prochaska, J. X., Chen, H.-W., et al. 2006, ApJ, 648, L930

Rau, A., Savaglio, S., Krühler, T., et al. 2010, ApJ, 720, 862

Savaglio, S. 2006, New J. Phys., 8, 195

Savaglio, S., Glazebrook, K., Crampton, D., et al. 2004, ApJ, 602, 51

Savaglio, S., Glazebrook, K., Le Borgne, D., et al. 2005, ApJ, 635, 260

Savaglio, S., Glazebrook, K., \& Le Borgne, D. 2009, ApJ, 691, 182

Sheffer, Y., Prochaska, J. X., Draine, B. T., Perley, D. A., \& Bloom, J. S. 2009, ApJ, 701, L63

Silva, A. I., \& Viegas, S. M. 2002, MNRAS, 329, 135

Schady, P., Page, M. J., Oates, et al. 2010, MNRAS, 401, 2773

Stratta, G., Maiolino, R., Fiore, F., \& D'Elia, V. 2007, ApJ, 661, 9

Sudilovsky, V., Savaglio, S., Vreeswijk, P., et al. 2007, ApJ, 669, 741

Tejos, N., Lopez, S., Prochaska, J. X., Chen, H.-W., \& Dessauges-Zavadsky, M. 2007, ApJ, 671, 622

Tejos, N., Lopez, S., Prochaska, J. X., et al. 2009, ApJ, 706, 1309

Thöne, C. C., Wiersema, K., Ledoux, C., et al. 2008, A\&A, 489, 37

Tumlinson, J., Prochaska, J. X., Chen, H.-W., Dessauges-Zavadsky, M., \& Bloom, J. S. 2007, ApJ, 668, 667

Uehara, T., Takahashi, H., \& McEnery, J. 2009, GCN Circ, 9934

van Dokkum, P. G. 2001, PASP, 113, 1420

Vergani, S. D., Petitjean, P., Ledoux, C., et al. 2009, A\&A, 503, 771

Vetere, L., Evans, P. A., \& Goad, M. R 2009, GCN Circ, 9936

Vetere, L. 2009, GCN 9961

Vreeswijk, P. M., Ellison, S. L., Ledoux, C., et al. 2004, A\&A, 419, 927

Vreeswijk, P. M., Ledoux, C., Smette, A., et al. 2007, A\&A, 468, 83

Zafar, T., Watson, D. J., Malesani, D., et al. 2010, A\&A, 515, 94 